\section{Guiding New Generations for Decades}

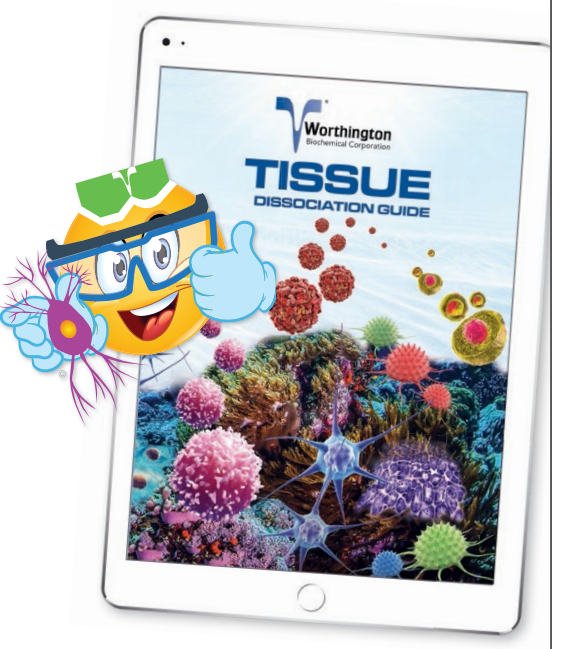

Worthington is supporting life science research and discovery, and we have done so for over 70 years.

Authoring technical manuals and guides both in print and digital formats. And, we are cited in thousands of respected scientific journals across the globe.

\section{Sharing Our Enzyme Expertise}

Order your free copy of the NEW Tissue Dissociation Guide, 18th Edition. Simply go to:

\section{Worthington-Biochem.com/} TissueDissociation/default.html

Come see us at EB '19 Booth 1044

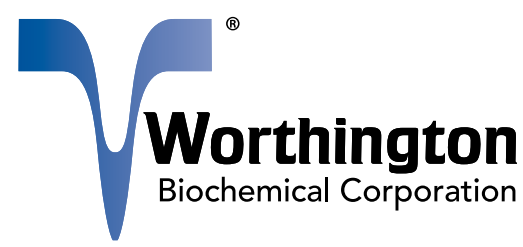

$800.445 .9603 \bullet 732.942 .1660$

\title{
Harnessing fungal bioluminescence
}

Researchers have developed the first fully genetically encodable eukaryotic bioluminescent system.

$\mathrm{B}$ oluminescence abounds in the natural world. Many organisms express luciferases, which are enzymes that catalyze the conversion of luciferin molecules to oxyluciferins-a reaction that is so energetically efficient that excess energy is emitted as light. Researchers use popular luciferases such as those from the firefly and the sea pansy (genus Renilla) as probes for molecular imaging because they give strong signal and do not require excitation light, unlike fluorescent proteins such as GFP.

One drawback to using luciferases as probes is that they require the addition of exogenous luciferins for signal generation. This can hamper biological studies, especially those involving living animals. Ilia Yampolsky from the Institute of Bioorganic Chemistry in Moscow, Russia, and a team of colleagues sought to address this limitation.

For the creation of a fully genetically encoded system, all of the enzymes required for synthesis of the the luciferin substrate must be known, along with the identity of the luciferase enzyme-a feat that has not been achieved for any eukaryotic luciferases. Yampolsky, who has studied light-emitting systems throughout his career, became interested in less widely studied luciferase systems, including those in fungi, noting that "the molecular basis of fungal bioluminescence has not been solved for more than 50 years despite several attempts; we took it as a challenge."

The identification of bioluminescencerelated genes in fungi was made possible by an earlier discovery of fungal luciferin by the same team. "Solving its structure was cumbersome and we spent several years working on it," recalls Yampolsky. "Then our colleagues in Krasnoyarsk suggested a crazy idea-to look for luciferin precursors in nonbioluminescent fungal species-and, surprisingly, it worked.'

The next step was identifying the luciferase gene from the fungus Neonothopanus nambi. This luciferase, nnLuz, has no described homologs and represents a novel protein family. Yampolsky recalls that this was a major challenge, noting that they were unable to identify the gene from a bacterial screen and that "it was only a year later that we identified the same gene by screening of cDNA libraries in yeasts." After discovery of the luciferase, identification of the full luciferin biosynthetic pathway

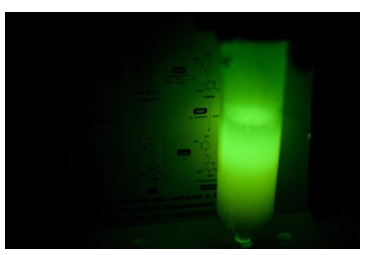

A photograph of $P$. pastoris expressing the bioluminescence genes from N. nambi. Reproduced from Kotlobay et al. (2018) with permission from the authors and PNAS as permitted under a CC BY-NC-ND license (https://creativecommons. org/licenses/by-nc-nd/4.0/legalcode).

was straightforward, as metabolic genes are typically clustered in fungal genomes.

The pathway involves four enzymes that can be expressed in a range of organisms to make them bioluminescent. Yampolsky notes that this development of autonomously glowing creatures is likely to be the main implication of the research. Indeed, the authors showed that they were able to generate bioluminescent Pichia pastoris by inducing expression of the necessary genes. Karen Sarkisyan, cofounder of Planta, a company that participated in the study, also highlights that the luciferin 3-hydroxyhispidin is stable and cell permeable, and that nnLuz gives signal comparable to that of firefly luciferase in mammalian systems.

Sarkisyan, who is driven by the idea of generating glowing plants, says he was most surprised by "the beauty and the simplicity of the fungal bioluminescent system." He says, "We hope that this system will bring bioluminescent technologies beyond being used just as tools in scientific research-for example, to create glowing plants for interior and landscape design, or perhaps even for street lighting. We also envision that the technology will enable development of new interfaces for life-life and life-computer communication based on light emission."

\section{Rita Strack}

Published online: 30 January 2019 https://doi.org/10.1038/s41592-019-0311-4

Research papers

Kotlobay, A. A. et al. Genetically encodable bioluminescent system from fungi. Proc. Natl Acad. Sci. USA 115, 12728-12732 (2018). 